\title{
FUNCTIONAL CALCULUS ON REAL INTERPOLATION SPACES FOR GENERATORS OF $C_{0}$-GROUPS
}

\author{
MARKUS HAASE AND JAN ROZENDAAL
}

\begin{abstract}
We study functional calculus properties of $C_{0}$-groups on real interpolation spaces, using transference principles. We obtain interpolation versions of the classical transference principle for bounded groups and of a recent transference principle for unbounded groups. Then we show that each group generator on a Banach space has a bounded $\mathrm{H}_{1}^{\infty}$-calculus on real interpolation spaces. Additional results are derived from this.
\end{abstract}

\section{INTRODUCTION}

The classical transference principle by Berkson, Gillespie and Muhly from [5] yields an estimate

$$
\left\|\int_{\mathbb{R}} U(s) x \mu(\mathrm{d} s)\right\|_{X} \leq M^{2}\left\|L_{\mu}\right\|_{\mathcal{L}\left(\mathrm{L}^{p}(X)\right)}\|x\|_{X}
$$

for all $x \in X$, where $(U(s))_{s \in \mathbb{R}} \subseteq \mathcal{L}(X)$ is a bounded $C_{0}$-group of operators on a Banach space $X$ with uniform bound $M, \mu$ is a complex Borel measure on $\mathbb{R}$ and $L_{\mu}$ is convolution with $\mu$ on $\mathrm{L}^{p}(X)$, the space of $p$-integrable $X$-valued functions, for $p \in[1, \infty]$. Under certain geometrical assumptions on $X$, the norm of $L_{\mu}$ can be bounded in terms of a suitable norm of the Fourier transform $\mathcal{F} \mu$ of $\mu$. For instance, if $X$ is a Hilbert space then $\left\|L_{\mu}\right\|_{\mathcal{L}\left(\mathrm{L}^{2}(X)\right)}$ is equal to $\|\mathcal{F} \mu\|_{\infty}$, by Plancherel's theorem. If $X$ is a UMD space and $p \in(1, \infty)$ then bounds for $\left\|L_{\mu}\right\|_{\mathcal{L}\left(\mathrm{L}^{p}(X)\right)}$ follow from the Mikhlin multiplier theorem. By combining this with (1.1), functional calculus bounds for the generator $A$ of $(U(s))_{s \in \mathbb{R}}$ can be obtained, i.e., estimates of the form $\|f(A)\| \leq C\|f\|_{F}$ for all $f$ in some function algebra $F$. Such bounds are important for evolution equations, conform for instance [2, 17.

Useful as this procedure is, the assumptions on the space $X$ restrict the generality of the results. In particular, Hilbert and UMD spaces are reflexive. Therefore the approach described above generally does not yield interesting results for groups of operators on non-reflexive spaces, such as $\mathrm{C}(K)$-spaces or $\mathrm{L}^{1}$-spaces. In this paper we take a different approach and consider transference principles on interpolation spaces. It is known that the functional calculus properties of operators improve upon restriction to interpolation spaces, conform for instance the result of Dore [6] that an invertible sectorial operator has a bounded sectorial $\mathrm{H}^{\infty}$-calculus on real interpolation spaces. However, we are interested in functional calculus on strips,

Date: September 4, 2018.

2010 Mathematics Subject Classification. 47A60, 47D03, 42B35, 42A45, 46B70.

Key words and phrases. Functional calculus, Transference, Operator semigroup, Fourier multiplier, Interpolation space.

The second-named author is supported by NWO-grant 613.000.908 "Applications of Transference Principles". 
the more natural choice for group generators. We use that on Besov spaces Fourier multiplier results hold that do not depend on the geometry of the underlying space 9.16. Since Besov spaces are obtained from real interpolation between $\mathrm{L}^{p}$ and Sobolev spaces, this fits into the setting of a transference principle on interpolation spaces. In Proposition 3.2 we derive the following version of (1.1) on the real interpolation space $(X, \mathrm{D}(A))_{\theta, q}$ from (1.4). For the $X$-valued Besov space $\mathrm{B}_{p, q}^{\theta}(X)$ see Section 2.2.

Proposition 1.1. Let $X$ be a Banach space and let $\theta \in(0,1), p \in[1, \infty)$ and $q \in[1, \infty]$. Then there exists a constant $C \geq 0$ such that the following holds. If $-\mathrm{i} A$ generate a $C_{0}$-group $(U(s))_{s \in \mathbb{R}}$ on a Banach space $X$ with $M:=\sup _{s \in \mathbb{R}}\|U(s)\|<$ $\infty$, then

$$
\left\|\int_{\mathbb{R}} U(s) x \mu(\mathrm{d} s)\right\|_{(X, \mathrm{D}(A))_{\theta, q}} \leq C M^{2}\left\|L_{\mu}\right\|_{\mathcal{L}\left(\mathrm{B}_{p, q}^{\theta}(X)\right)}\|x\|_{(X, \mathrm{D}(A))_{\theta, q}}
$$

for all complex Borel meaures $\mu$ on $\mathbb{R}$ and $x \in(X, \mathrm{D}(A))_{\theta, q}$.

Combining Proposition 1.1 with the aforementioned Fourier multiplier results on Besov spaces yields the following, a consequence of Corollary 3.5 .

Corollary 1.2. Let $-\mathrm{i} A$ generate a uniformly bounded $C_{0}$-group $(U(s))_{s \in \mathbb{R}}$ on a Banach space $X$, and let $\theta \in(0,1), q \in[1, \infty]$. Then there exists a constant $C \geq 0$ such that

$$
\left\|\int_{\mathbb{R}} U(s) x \mu(\mathrm{d} s)\right\|_{(X, \mathrm{D}(A))_{\theta, q}} \leq C \sup _{s \in \mathbb{R}}|\mathcal{F} \mu(s)|+(1+|s|)\left|(\mathcal{F} \mu)^{\prime}(s)\right|\|x\|_{(X, \mathrm{D}(A))_{\theta, q}}
$$

for all $x \in(X, \mathrm{D}(A))_{\theta, q}$ and for each $\mu \in \mathrm{M}(\mathbb{R})$ such that $\mathcal{F} \mu \in \mathrm{C}^{1}(\mathbb{R})$ with $\sup _{s \in \mathbb{R}}(1+|s|)\left|(\mathcal{F} \mu)^{\prime}(s)\right|<\infty$.

We also obtain an interpolation version of the transference principle for unbounded groups from 12, as Proposition 3.1. In terms of functional calculus for the part of $A$ in $(X, \mathrm{D}(A))_{\theta, q}$, these transference principles yield a result for functions in the analytic Mikhlin algebra

$$
\mathrm{H}_{1}^{\infty}\left(\mathrm{St}_{\omega}\right):=\left\{f \in \mathrm{H}^{\infty}\left(\mathrm{St}_{\omega}\right)\left|\sup _{z \in \mathrm{St}_{\omega}}(1+|z|)\right| f^{\prime}(z) \mid<\infty\right\}
$$

endowed with the norm

$$
\|f\|_{\mathrm{H}_{1}^{\infty}\left(\mathrm{St}_{\omega}\right)}:=\sup _{z \in \mathrm{St}_{\omega}}|f(z)|+(1+|z|)\left|f^{\prime}(z)\right| \quad\left(f \in \mathrm{H}_{1}^{\infty}\left(\mathrm{St}_{\omega}\right)\right) .
$$

Here $\mathrm{St}_{\omega}:=\{z \in \mathbb{C}|| \operatorname{Im}(z) \mid<\omega\}$ for $\omega>0$. Note that definition (1.3) of the norm in the analytic Mikhlin algebra is different from that in 12, where the quantity $\|f\|=\sup _{z \in \mathrm{St}_{\omega}}|f(z)|+\left|z f^{\prime}(z)\right|$ is considered. However, the two norms are equivalent on domains containing zero, and (1.3) is more natural in the setting of transference principles on (inhomogeneous) Besov spaces, since Fourier multiplier results on such spaces require an inhomogeneous condition at zero. See also Remarks 3.7 and 4.2

Our main functional calculus result is as follows. For the group type $\theta(U)$ see (2.1), and for a proof of this result see Theorem 4.1.

Theorem 1.3. Let $-\mathrm{i} A$ be the generator of a $C_{0}$-group $(U(s))_{s \in \mathbb{R}}$ on a Banach space $X$, and let $\theta \in(0,1), q \in[1, \infty]$. Then the part of $A$ in $(X, \mathrm{D}(A))_{\theta, q}$ has a bounded $\mathrm{H}_{1}^{\infty}\left(\mathrm{St}_{\omega}\right)$-calculus for all $\omega>\theta(U)$. If $(U(s))_{s \in \mathbb{R}}$ is uniformly bounded then the constant bounding the $\mathrm{H}_{1}^{\infty}\left(\mathrm{St}_{\omega}\right)$-calculus does not depend on $\omega>0$. 
In [12, Theorem 1.3] is obtained for group generators on UMD spaces and functional calculus for the operator $A$ itself. Our result shows that on interpolation spaces no assumptions on the geometry of the underlying space are required. This means that even on spaces which are not UMD, such as $\mathrm{C}(K)$-spaces and $\mathrm{L}^{1}$-spaces, one can obtain functional calculus results if one is willing to restrict to interpolation spaces. Moreover, our results reaffirm the philosophy that the functional calculus properties of an operator improve when restricted to interpolation spaces, as was already evidenced for functions on sectors by the result of Dore [6. Theorem 3.2].

From Theorem 1.3 we deduce other functional calculus statements, for sectorial operators and generators of cosine functions.

Section 2 provides the necessary background on functional calculus and the theory of Fourier multipliers on Besov spaces. In Section 3 we establish transference principles on interpolation spaces, and in Section 4 we prove Theorem 1.3. Section 5 contains additional results that can be derived from this.

1.1. Notation and terminology. The natural numbers are $\mathbb{N}:=\{1,2, \ldots\}$, and we write $\mathbb{N}_{0}:=\mathbb{N} \cup\{0\}$. The letters $X$ and $Y$ denote Banach spaces over the complex number field, and $\mathcal{L}(X)$ is the Banach algebra of all bounded operators on $X$. The domain $\mathrm{D}(A) \subseteq X$ of a closed unbounded operator $A$ on $X$ is a Banach space when endowed with the norm

$$
\|x\|_{\mathrm{D}(A)}:=\|x\|+\|A x\| \quad(x \in \mathrm{D}(A)) .
$$

The spectrum of $A$ is $\sigma(A)$ and the resolvent set $\rho(A):=\mathbb{C} \backslash \sigma(A)$. For $z \in \rho(A)$ the operator $R(z, A):=(z \mathrm{I}-A)^{-1} \in \mathcal{L}(X)$ is the resolvent of $A$ at $z$.

For $p \in[1, \infty], \mathrm{L}^{p}(\mathbb{R} ; X)$ is the Bochner space of equivalence classes of $X$-valued Lebesgue $p$-integrable functions on $\mathbb{R}$. The Hölder conjugate of $p \in[1, \infty]$ is $p^{\prime}$ and is defined by $\frac{1}{p}+\frac{1}{p^{\prime}}=1$. The norm on $\mathrm{L}^{p}(\mathbb{R} ; X)$ is usually denoted by $\|\cdot\|_{p}$. In the case $X=\mathbb{C}$ we will simply write $\mathrm{L}^{p}(\mathbb{R})=\mathrm{L}^{p}(\mathbb{R} ; \mathbb{C})$.

By $\mathrm{M}(\mathbb{R})$ we denote the space of complex-valued Borel measures on $\mathbb{R}$ with the total variation norm. For $\omega \geq 0$ we let $\mathrm{M}_{\omega}(\mathbb{R})$ consist of those $\mu \in \mathrm{M}(\mathbb{R})$ of the form $\mu(\mathrm{d} s)=\mathrm{e}^{-\omega|s|} \nu(\mathrm{d} s)$ for some $\nu \in \mathrm{M}(\mathbb{R})$, with

$$
\|\mu\|_{\mathrm{M}_{\omega}(\mathbb{R})}:=\left\|\mathrm{e}^{\omega|\cdot|} \mu\right\|_{\mathrm{M}(\mathbb{R})} .
$$

Note that $\mathrm{M}_{\omega}(\mathbb{R})$ is a Banach algebra under convolution. A function $g$ such that $\left[s \mapsto g(s) \mathrm{e}^{\omega|s|}\right] \in \mathrm{L}^{1}(\mathbb{R})$ is usually identified with its associated measure $\mu \in \mathrm{M}_{\omega}(\mathbb{R})$ given by $\mu(\mathrm{d} s)=g(s) \mathrm{d} s$.

For $\Omega \neq \emptyset$ open in $\mathbb{C}$ we let $H^{\infty}(\Omega)$ be the unital Banach algebra of bounded holomorphic functions on $\Omega$ with the supremum norm

$$
\|f\|_{\mathrm{H}^{\infty}(\Omega)}:=\sup _{z \in \Omega}|f(z)| \quad\left(f \in \mathrm{H}^{\infty}(\Omega)\right) .
$$

We mainly consider the case where $\Omega$ is a strip of the form

$$
\mathrm{St}_{\omega}:=\{z \in \mathbb{C}|| \operatorname{Im}(z) \mid<\omega\}
$$

for $\omega>0$, with $\mathrm{St}_{0}:=\mathbb{R}$.

The Schwartz class $\mathcal{S}(\mathbb{R} ; X)$ is the space of $X$-valued rapidly decreasing smooth functions on $\mathbb{R}$, and the space of $X$-valued tempered distributions is $\mathcal{S}^{\prime}(\mathbb{R} ; X)$. The Fourier transform of an $X$-valued tempered distribution $\Phi \in \mathcal{S}^{\prime}(\mathbb{R} ; X)$ is denoted 
by $\mathcal{F} \Phi$. For instance, if $\mu \in \mathrm{M}_{\omega}(\mathbb{R})$ for $\omega>0$ then $\mathcal{F} \mu \in \mathrm{H}^{\infty}\left(\mathrm{St}_{\omega}\right) \cap \mathrm{C}\left(\overline{\mathrm{St}_{\omega}}\right)$ is given by

$$
\mathcal{F} \mu(z):=\int_{\mathbb{R}} \mathrm{e}^{-\mathrm{i} s z} \mu(\mathrm{d} s) \quad\left(z \in \mathrm{St}_{\omega}\right) .
$$

If $X$ and $Y$ are Banach spaces that are embedded continuously into a Hausdorff topological vector space $Z$, then we call $(X, Y)$ an interpolation couple. We let

$$
K(t, z):=\inf \left\{\|x\|_{X}+t\|y\|_{Y} \mid x \in X, y \in Y, x+y=z\right\}
$$

for $t>0$ and $z \in X+Y \subseteq Z$. The real interpolation space of $X$ and $Y$ with parameters $\theta \in[0,1]$ and $q \in[1, \infty]$ is

$$
(X, Y)_{\theta, q}:=\left\{z \in X+Y \mid\left[t \mapsto t^{-\theta} K(t, z)\right] \in \mathrm{L}^{q}((0, \infty), \mathrm{d} t / t)\right\},
$$

a Banach space when equipped with the norm

$$
\|z\|_{(X, Y)_{\theta, q}}:=\left\|t \mapsto t^{-\theta} K(t, z)\right\|_{L^{q}((0, \infty), \mathrm{d} t / t)} \quad\left(z \in(X, Y)_{\theta, q}\right) .
$$

If $T: X+Y \rightarrow X+Y$ restricts to a bounded operator on $X$ and a bounded operator on $Y$ then

$$
\|T\|_{\mathcal{L}\left((X, Y)_{\theta, q}\right)} \leq\|T\|_{\mathcal{L}(X)}^{1-\theta}\|T\|_{\mathcal{L}(Y)}^{\theta}
$$

for all $\theta \in(0,1)$ and $q \in[1, \infty]$, Theorem 3.1.2]. We mainly consider interpolation spaces for the couple $(X, \mathrm{D}(A))$, where $A$ is a closed operator on $X$. We write

$$
\mathrm{D}_{A}(\theta, q):=(X, \mathrm{D}(A))_{\theta, q}
$$

and

$$
\|x\|_{\theta, q}:=\|x\|_{\mathrm{D}_{A}(\theta, q)} \quad\left(x \in \mathrm{D}_{A}(\theta, q)\right) .
$$

For an operator $B$ on $X$ and a continuously embedded space $Y \hookrightarrow X$, the operator $B_{Y}$ on $Y$ that satisfies $B_{Y} y=B y$ for elements in its domain

$$
\mathrm{D}\left(B_{Y}\right):=\{y \in \mathrm{D}(B) \cap Y \mid B y \in Y\}
$$

is the part of $B$ in $Y$. If $Y=\mathrm{D}_{A}(\theta, q)$ for $\theta \in(0,1)$ and $q \in[1, \infty]$ then we write

$$
B_{\theta, q}:=B_{\mathrm{D}_{A}(\theta, q)} \text {. }
$$

Throughout, an $X$-valued function space $\Phi(\mathbb{R} ; X)$ on the real line will be denoted by $\Phi(X)$ whenever little confusion can arise.

\section{Functional CALCulus And Fourier multipliers}

2.1. Functional calculus. We assume that the reader is familiar with the basics of the theory of $C_{0}$-groups as developed in, for instance, 8 , and merely recall some of the notions and results in functional calculus theory that are used. Details on functional calculus for group generators can be found in [10, Chapter 4].

Let $-\mathrm{i} A$ be the generator of a $C_{0}$-group $(U(s))_{s \in \mathbb{R}}$ on a Banach space $X$. Then the group type of $U$,

$$
\theta(U):=\inf \left\{\omega \geq 0 \mid \exists M \geq 1 \text { such that }\|U(s)\| \leq M \mathrm{e}^{\omega|s|} \text { for all } s \geq 0\right\},
$$

is finite. Moreover, $A$ is a strip-type operator of height $\omega_{0}:=\theta(U)$, i.e., $\sigma(A) \subseteq \overline{\mathrm{St}_{\omega_{0}}}$ and

$$
\sup _{\lambda \in \mathbb{C} \backslash \mathrm{St}_{\omega}}\|R(\lambda, A)\|<\infty \quad \text { for all } \omega>\omega_{0}
$$


The strip-type functional calculus for $A$ is defined as follows. First, operators $f(A) \in$ $\mathcal{L}(X)$ are associated with functions

$$
f \in \mathcal{E}\left(\mathrm{St}_{\omega}\right):=\left\{g \in \mathrm{H}^{\infty}\left(\mathrm{St}_{\omega}\right) \mid g(z) \in O\left(|z|^{-\alpha}\right) \text { for some } \alpha>1 \text { as }|\operatorname{Re}(z)| \rightarrow \infty\right\}
$$

for $\omega>\omega_{0}$, by a Cauchy-type integral

$$
f(A):=\frac{1}{2 \pi \mathrm{i}} \int_{\delta \mathrm{St}_{\omega^{\prime}}} f(z) R(z, A) \mathrm{d} z .
$$

Here $\delta \mathrm{St}_{\omega^{\prime}}$ is the positively oriented boundary of $\mathrm{St}_{\omega^{\prime}}$ for $\omega^{\prime} \in\left(\omega_{0}, \omega\right)$. This procedure is independent of the choice of $\omega^{\prime}$ by Cauchy's theorem, and yields an algebra homomorphism $\mathcal{E}\left(\mathrm{St}_{\omega}\right) \rightarrow \mathcal{L}(X)$. The definition of $f(A)$ is extended to a larger class of functions by regularization, i.e.

$$
f(A):=e(A)^{-1}(e f)(A)
$$

if there exists $e \in \mathcal{E}\left(\mathrm{St}_{\omega}\right)$ with $e(A)$ injective and ef $\in \mathcal{E}\left(\mathrm{St}_{\omega}\right)$. This yields a closed unbounded operator $f(A)$ on $X$, and the definition of $f(A)$ is independent of the choice of the regularizer $e$. The algebra of all meromorphic functions on $\mathrm{St}_{\omega}$ that are regularizable for $A$ is denoted by $\mathcal{M}_{A}\left(\mathrm{St}_{\omega}\right)$. Each $f \in \mathrm{H}^{\infty}\left(\mathrm{St}_{\omega}\right)$ is regularizable by the function $z \mapsto(\lambda-z)^{-2}$, for $|\operatorname{Im}(\lambda)|>\omega$.

Since $-\mathrm{i} A$ generates a $C_{0}$-group, the Hille-Phillips functional calculus for $A$ yields certain functions $f$ that give rise to bounded operators $f(A)$. Fix $M \geq 1$ and $\omega \geq 0$ such that $\|U(s)\| \leq M \mathrm{e}^{\omega|s|}$ for all $s \in \mathbb{R}$. For $\mu \in \mathrm{M}_{\omega}(\mathbb{R})$ define

$$
U_{\mu} x:=\int_{\mathbb{R}} U(s) x \mu(\mathrm{d} s) \quad(x \in X) .
$$

Then $\mu \mapsto U_{\mu}$ is an algebra homomorphism $\mathrm{M}_{\omega}(\mathbb{R}) \rightarrow \mathcal{L}(X)$. The following lemma, Lemma 2.2 in [11, shows that the Hille-Phillips calculus extends the strip-type calculus for $A$.

Lemma 2.1. Let $X, A$ and $U$ be as above, and let $\omega^{\prime}>\omega \geq 0$.

a) For each $f \in \mathcal{E}\left(\mathrm{St}_{\omega^{\prime}}\right)$ there exists $\mu \in \mathrm{M}_{\omega}(\mathbb{R})$ such that $f=\mathcal{F} \mu$.

b) Let $\mu \in \mathrm{M}_{\omega}(\mathbb{R})$ be such that $\mathcal{F} \mu$ extends to an element of $\mathcal{M}_{A}\left(\mathrm{St}_{\omega^{\prime}}\right)$. Then $f(A)=U_{\mu} \in \mathcal{L}(X)$ and

$$
\sup _{t \in \mathbb{R}}\|f(t+A)\| \leq M\|\mu\|_{\mathrm{M}_{\omega}(\mathbb{R})} .
$$

We now consider functional calculus for operators on interpolation spaces. The following lemma shows that, in particular, the functional calculi for $A$ and $A_{\theta, q}$ are compatible.

Lemma 2.2. Let $A$ be a strip-type operator of height $\omega_{0}$ on a Banach space $X$ and let $\theta \in(0,1), q \in[1, \infty]$ and $m, n \in \mathbb{N}_{0}$. Let $Y:=\left(\mathrm{D}\left(A^{m}\right), \mathrm{D}\left(A^{n}\right)\right)_{\theta, q}$.

a) The part $A_{Y}$ of $A$ in $Y$ is a strip-type operator of height $\omega_{0}$. Moreover, $f \in \mathcal{M}_{A_{Y}}\left(\mathrm{St}_{\omega}\right)$ with $f\left(A_{Y}\right)=f(A)_{Y}$ for all $\omega>\omega_{0}$ and $f \in \mathcal{M}_{A}\left(\mathrm{St}_{\omega}\right)$.

b) If $-\mathrm{i} A$ generates a $C_{0}$-group $(U(s))_{s \in \mathbb{R}}$ on $X$ and $q<\infty$, then $-\mathrm{i} A_{Y}$ generates the $C_{0}$-group $\left(U(s)_{Y}\right)_{s \in \mathbb{R}}$. In particular, $\mathrm{D}\left(A_{Y}\right)$ is dense in $Y$.

Proof. a) First note that, for all $k \in \mathbb{N}_{0}$ and $\lambda \in \rho(A), R(\lambda, A)$ leaves $\mathrm{D}\left(A^{k}\right)$ invariant with $\|R(\lambda, A)\|_{\mathcal{L}\left(\mathrm{D}\left(A^{k}\right)\right)} \leq\|R(\lambda, A)\|_{\mathcal{L}(X)}$. By (1.5), $R(\lambda, A)$ leaves $Y$ invariant with

$$
\|R(\lambda, A)\|_{\mathcal{L}(Y)} \leq\|R(\lambda, A)\|_{\mathcal{L}(X)} .
$$


By [10, Proposition A.2.8], $\sigma\left(A_{Y}\right) \subseteq \sigma(A)$ and $R\left(\lambda, A_{Y}\right)=R(\lambda, A)_{Y}$ for all $\lambda \in$ $\rho(A)$. Hence (2.3) yields the first statement. Let $\omega>\omega_{0}$ and $f \in \mathcal{E}\left(\mathrm{St}_{\omega}\right)$ be given. Then

$$
f\left(A_{Y}\right) y=\frac{1}{2 \pi \mathrm{i}} \int_{\Gamma} f(z) R\left(z, A_{Y}\right) y \mathrm{~d} z=\frac{1}{2 \pi \mathrm{i}} \int_{\Gamma} f(z) R(z, A) y \mathrm{~d} z=f(A) y
$$

for some contour $\Gamma$ and all $y \in Y$. For a general $f \in \mathcal{M}_{A}\left(\mathrm{St}_{\omega}\right)$, note that $e$ is a regulariser for $f$ in the functional calculus for $A_{Y}$ if it is a regulariser for $f$ in the functional calculus for $A$, since then $e\left(A_{Y}\right)=e(A)_{Y}$ is injective. The rest follows by regularization.

b) By (1.5), $\left\|U(s)_{Y}\right\| \leq\|U(s)\|$ for all $s \in \mathbb{R}$. Hence $\left(U(s)_{Y}\right)_{s \in \mathbb{R}}$ is locally bounded. Since it is strongly continuous on the dense subset $\mathrm{D}\left(A^{\max (n, m)}\right) \subseteq Y$ [19. Proposition 1.2.5], it is strongly continuous on $Y$. By [8, p. 60], $-\mathrm{i} A_{Y}$ is its generator.

Remark 2.3. Part b) of Lemma 2.2 ensures that the integral in (2.2) is well-defined and converges in $\mathrm{D}_{A}(\theta, q)$, for $x \in \mathrm{D}_{A}(\theta, q)$ and $q<\infty$. Even though $(U(s))_{s \in \mathbb{R}}$ is not strongly continuous on $\mathrm{D}_{A}(\theta, \infty)$ in general, the integral is well-defined and converges in $X$. Since $\mathrm{D}_{A}(\theta, q)$ is continuously embedded in $X$ for all $\theta \in(0,1)$ and $q \in[1, \infty]$, the value of the integral does not depend on the space in which convergence takes place. Hence from now on we regularly will not specify in which norm (2.2) converges.

Let $A$ be a strip-type operator of height $\omega_{0}$ and $\omega>\omega_{0}$. For a Banach algebra $F$ of functions that is continuously embedded in $\mathrm{H}^{\infty}\left(\mathrm{St}_{\omega}\right)$, we say that $A$ has a bounded $F$-calculus if there exists a constant $C \geq 0$ such that $f(A) \in \mathcal{L}(X)$ with

$$
\|f(A)\|_{\mathcal{L}(X)} \leq C\|f\|_{F} \quad \text { for all } f \in F .
$$

The next lemma from [10, Proposition 5.1.7] is fundamental.

Lemma 2.4 (Convergence Lemma). Let $A$ be a densely defined strip-type operator of height $\omega_{0}$ on a Banach space $X$. Let $\omega>\omega_{0}$ and $\left(f_{j}\right)_{j \in J} \subseteq \mathrm{H}^{\infty}\left(\mathrm{St}_{\omega}\right)$ be a net satisfying the following conditions:

(1) $\sup _{j \in J}\left\|f_{j}\right\|_{\mathrm{H}^{\infty}\left(\mathrm{St}_{\omega}\right)}<\infty$;

(2) $f(z):=\lim _{j} f_{j}(z)$ exists for all $z \in \mathrm{St}_{\omega}$;

(3) $\sup _{j \in J}\left\|f_{j}(A)\right\|_{\mathcal{L}(X)}<\infty$.

Then $f \in \mathrm{H}^{\infty}\left(\mathrm{St}_{\omega}\right), f(A) \in \mathcal{L}(X), f_{j}(A) \rightarrow f(A)$ strongly and

$$
\|f(A)\| \leq \limsup _{j \in J}\left\|f_{j}(A)\right\| .
$$

2.2. Fourier multipliers on Besov spaces. Let us summarize some results about Fourier multipliers on vector-valued Besov spaces which will be used later on. Details can be found in [1] and [9].

Let $\psi \in \mathrm{C}^{\infty}(\mathbb{R})$ be a nonnegative function with support in $\left[\frac{1}{2}, 2\right]$ such that

$$
\sum_{k=-\infty}^{\infty} \psi\left(2^{-k} s\right)=1 \quad \text { for all } s \in(0, \infty) .
$$

For $k \in \mathbb{N}$ and $s \in \mathbb{R}$ let $\varphi_{k}(s):=\psi\left(2^{-k}|s|\right)$, and let $\varphi_{0}(s):=1-\sum_{k=1}^{\infty} \varphi_{k}(s)$. Let $X$ be a Banach space and let $p, q \in[1, \infty]$ and $r \in \mathbb{R}$ be given. The (inhomogeneous) Besov space $\mathrm{B}_{p, q}^{r}(\mathbb{R} ; X)$ consists of all $X$-valued tempered distributions 
$f \in \mathcal{S}^{\prime}(\mathbb{R} ; X)$ such that

$$
\|f\|_{\mathrm{B}_{p, q}^{r}(\mathbb{R} ; X)}:=\left\|\left(2^{k r}\left\|\mathcal{F}^{-1} \varphi_{k} * f\right\|_{L^{p}(\mathbb{R} ; X)}\right)_{k=0}^{\infty}\right\|_{\ell^{q}}<\infty,
$$

endowed with the norm $\|f\|_{\mathrm{B}_{p, q}^{r}(\mathbb{R} ; X)}$. Then $\mathrm{B}_{p, q}^{r}(\mathbb{R} ; X)$ is a Banach space such that $\mathcal{S}(\mathbb{R} ; X) \subseteq \mathrm{B}_{p, q}^{r}(\mathbb{R} ; X)$, and a different choice of $\psi$ leads to an equivalent norm on $\mathrm{B}_{p, q}^{r}(\mathbb{R} ; X)$.

For $n \in \mathbb{N}$ and $p \in[1, \infty]$ the Sobolev space

$$
\mathrm{W}^{n, p}(\mathbb{R} ; X):=\left\{f \in \mathrm{L}^{p}(\mathbb{R} ; X) \mid f^{(k)} \in \mathrm{L}^{p}(\mathbb{R} ; X) \text { for all } 1 \leq k \leq n\right\},
$$

is a Banach space when endowed with the norm

$$
\|f\|_{n, p}:=\|f\|_{\mathrm{W}^{n, p}(X)}:=\|f\|_{p}+\left\|f^{(n)}\right\|_{p} \quad\left(f \in \mathrm{W}^{n, p}(\mathbb{R} ; X)\right) .
$$

In the case $X=\mathbb{C}$ we simply write $\mathrm{W}^{n, p}(\mathbb{R})=\mathrm{W}^{1, p}(\mathbb{R} ; \mathbb{C})$.

The following lemma is equation (5.9) in [1. The fact that the constant $C$ does not depend on the particular Banach space follows from a direct sum argument.

Lemma 2.5. Let $\theta \in(0,1), p \in[1, \infty), q \in[1, \infty]$ and $n \in \mathbb{N}$. Then there exists a constant $C>0$ such that, for any Banach space $X,\left(\mathrm{~L}^{p}(X), \mathrm{W}^{n, p}(X)\right)_{\theta, q}=\mathrm{B}_{p, q}^{n \theta}(X)$ with

$$
\frac{1}{C}\|f\|_{\mathrm{B}_{p, q}^{n \theta}(X)} \leq\|f\|_{\left(\mathrm{L}^{p}(X), \mathrm{W}^{n, p}(X)\right)_{\theta, q}} \leq C\|f\|_{\mathrm{B}_{p, q}^{n \theta}(X)} \quad\left(f \in \mathrm{B}_{p, q}^{n \theta}(X)\right) .
$$

Let $m \in \mathrm{L}^{\infty}(\mathbb{R} ; \mathcal{L}(X)), p, q \in[1, \infty]$ and $r \in \mathbb{R}$. We say that $m$ is a bounded Fourier multiplier on $\mathrm{B}_{p, q}^{r}(X)$ if there is a unique bounded operator $T_{m}: \mathrm{B}_{p, q}^{r}(X) \rightarrow$ $\mathrm{B}_{p, q}^{r}(X)$ such that

$$
T_{m}(f)=\mathcal{F}^{-1}(m \cdot \mathcal{F} f)
$$

for all $f \in \mathcal{S}(X)$. Each $\mu \in \mathrm{M}(\mathbb{R})$ induces a bounded Fourier multiplier $\mathcal{F} \mu$ with

$$
T_{\mathcal{F} \mu}(f)=L_{\mu}(f):=\mu * f \quad(f \in \mathcal{S}(X)) .
$$

The main result about Fourier multipliers on Besov spaces that we use is the following, Corollary 4.15 from [9].

Proposition 2.6. There exists a constant $C \geq 0$ such that the following holds. Let $X$ be a Banach space, $p, q \in[1, \infty]$ and $r \in \mathbb{R}$. If $m: \mathbb{R} \rightarrow \mathbb{C}$ is such that $\varphi_{k} m \in \mathrm{B}_{2,1}^{1 / 2}(\mathbb{R} ; \mathbb{C})$ for all $k \in \mathbb{N}_{0}$, and

$$
M:=\sup _{k \in \mathbb{N}_{0}} \inf _{a>0}\left\|\left(\varphi_{k} m\right)(a \cdot)\right\|_{\mathrm{B}_{2,1}^{1 / 2}(\mathbb{R} ; \mathbb{C})}<\infty,
$$

then $m$ is a bounded Fourier multiplier on $\mathrm{B}_{p, q}^{r}(X)$ with $\left\|T_{m}\right\|_{\mathcal{L}\left(\mathrm{B}_{p, q}^{r}(X)\right)} \leq C M$.

Corollary 2.7. There exists a constant $C \geq 0$ such that for all Banach spaces $X$, $p, q \in[1, \infty], r \in \mathbb{R}$ and all $m \in \mathrm{C}^{1}(\mathbb{R} ; \mathbb{C})$ with

$$
N:=\sup _{s \in \mathbb{R}}|m(s)|+(1+|s|)\left|m^{\prime}(s)\right|<\infty,
$$

$m$ is a bounded Fourier multiplier on $\mathrm{B}_{p, q}^{r}(X)$ with $\left\|T_{m}\right\|_{\mathcal{L}\left(\mathrm{B}_{p, q}^{r}(X)\right)} \leq C N$.

Proof. This follows as in [9, Corollary 4.11]. See also [9, Remark 4.16]. 


\section{TRANSFERENCE PRINCIPLES}

3.1. Unbounded groups. We first establish an interpolation version of the transference principle for unbounded groups from [12. Note that, for each $\mu \in \mathrm{M}(\mathbb{R})$ and $p \in[1, \infty]$, the convolution operator $L_{\mu}$ from (2.5) extends to a bounded operator on $\mathrm{L}^{p}(X)$, by Young's inequality. For $\omega \geq 0$ and $\mu \in \mathrm{M}_{\omega}(\mathbb{R})$ let $\mu_{\omega} \in \mathrm{M}(\mathbb{R})$ be given by $\mu_{\omega}(\mathrm{d} s):=\cosh (\omega s) \mu(\mathrm{d} s)$.

Proposition 3.1. Let $0 \leq \omega_{0}<\omega, \theta \in(0,1), p \in[1, \infty)$ and $q \in[1, \infty]$. Then there exists a constant $C \geq 0$ such that the following holds. If $-\mathrm{i} A$ generates a $C_{0}$-group $(U(s))_{s \in \mathbb{R}}$ on a Banach space $X$ such that $\|U(s)\|_{\mathcal{L}(X)} \leq M \cosh \left(\omega_{0} s\right)$ for all $s \in \mathbb{R}$ and some $M \geq 1$, then

$$
\left\|\int_{\mathbb{R}} U(s) x \mu(\mathrm{d} s)\right\|_{\theta, q} \leq C M^{2}\left\|L_{\mu_{\omega}}\right\|_{\mathcal{L}\left(\mathrm{B}_{p, q}^{\theta}(X)\right)}\|x\|_{\theta, q}
$$

for all $\mu \in \mathrm{M}_{\omega}(\mathbb{R})$ and $x \in \mathrm{D}_{A}(\theta, q)$.

Proof. Let $\mu \in \mathrm{M}_{\omega}(\mathbb{R})$ be given and let $U_{\mu}$ be as in (2.2). By the proof of Theorem 3.2 in [12, we can factorize $U_{\mu}$ as $U_{\mu}=P \circ L_{\mu_{\omega}} \circ \iota$, where

- $\iota: X \rightarrow \mathrm{L}^{p}(X)$ is given by

$$
\iota x(s):=\psi(-s) U(-s) x \quad(x \in X, s \in \mathbb{R}),
$$

with

$$
\psi(s):=\frac{1}{\cosh (\alpha s)} \quad(s \in \mathbb{R})
$$

for $\alpha>\omega$ fixed.

- $P: \mathrm{L}^{p}(X) \rightarrow X$ is given by

$$
P f:=\int_{\mathbb{R}} \varphi(s) U(s) f(s) \mathrm{d} s \quad\left(f \in \mathrm{L}^{p}(X)\right),
$$

with

$$
\varphi(s):=\frac{\sqrt{8} \omega}{\pi} \frac{\cosh (\omega s)}{\cosh (2 \omega s)} \quad(s \in \mathbb{R}) .
$$

Then, using Hölder's inequality,

$$
\begin{aligned}
\|\iota\|_{\mathcal{L}\left(X, \mathrm{~L}^{p}(X)\right)} & \leq M\left\|\psi \cosh \left(\omega_{0} \cdot\right)\right\|_{p}, \\
\|P\|_{\mathcal{L}\left(\mathrm{L}^{p}(X), X\right)} & \leq M\left\|\varphi \cosh \left(\omega_{0} \cdot\right)\right\|_{p^{\prime}} .
\end{aligned}
$$

We claim that $\iota: \mathrm{D}(A) \rightarrow \mathrm{W}^{1, p}(X)$ and $P: \mathrm{W}^{1, p}(X) \rightarrow \mathrm{D}(A)$ are well-defined and bounded. To prove this claim, first let $x \in \mathrm{D}(A)$. Then $\iota x \in \mathrm{C}^{1}(\mathbb{R})$ with

$$
\begin{aligned}
(\iota x)^{\prime}(s) & =-\psi^{\prime}(-s) U(-s) x+\mathrm{i} \psi(-s) U(-s) A x \\
& =-\alpha \frac{\tanh (\alpha s)}{\cosh (\alpha s)} U(-s) x+\mathrm{i} \frac{1}{\cosh (\alpha s)} U(-s) A x
\end{aligned}
$$

for all $s \in \mathbb{R}$. Hence $(\iota x)^{\prime} \in \mathrm{L}^{p}(\mathbb{R})$ with

$$
\left\|(\iota x)^{\prime}\right\|_{p} \leq \alpha M\|\tanh \|_{L^{\infty}(\mathbb{R})}\left\|\frac{\cosh \left(\omega_{0} \cdot\right)}{\cosh (\alpha \cdot)}\right\|_{p}\|x\|_{X}+M\left\|\frac{\cosh \left(\omega_{0} \cdot\right)}{\cosh (\alpha \cdot)}\right\|_{p}\|A x\|_{X} .
$$


Combining this with (3.1) implies that $\iota x \in \mathrm{W}^{1, p}(\mathbb{R})$ with

$$
\|\iota x\|_{1, p} \leq M\left(\alpha\|\tanh \|_{\mathrm{L}^{\infty}(\mathbb{R})}+1\right)\left\|\frac{\cosh \left(\omega_{0} \cdot\right)}{\cosh (\alpha \cdot)}\right\|_{p}\|x\|_{\mathrm{D}(A)} .
$$

This shows that $\iota: \mathrm{D}(A) \rightarrow \mathrm{W}^{1, p}(X)$ is bounded. To prove the claim for $P$, fix $f \in \mathcal{S}(X)$ and note that

$$
\frac{1}{h}(U(h)-\mathrm{I}) P f=\int_{\mathbb{R}} U(s) \frac{\varphi(s-h) f(s-h)-\varphi(s) f(s)}{h} \mathrm{~d} s
$$

for $h>0$. The latter expression converges to $-\int_{\mathbb{R}} U(s)(\varphi f)^{\prime}(s) \mathrm{d} s \in X$ as $h \rightarrow 0$, by the dominated convergence theorem. Hence $P f \in \mathrm{D}(A)$ with

$$
A P f=\lim _{h \rightarrow 0} \frac{1}{h}(U(h)-\mathrm{I}) P f=-\int_{\mathbb{R}} U(s)\left(\varphi^{\prime}(s) f(s)+\varphi(s) f^{\prime}(s)\right) \mathrm{d} s .
$$

Another application of Hölder's inequality yields

$$
\|A P f\|_{X} \leq M\left\|\varphi^{\prime} \cosh \left(\omega_{0} \cdot\right)\right\|_{p^{\prime}}\|f\|_{p}+M\left\|\varphi \cosh \left(\omega_{0} \cdot\right)\right\|_{p^{\prime}}\left\|f^{\prime}\right\|_{p} .
$$

Combining this with (3.2) implies

$$
\|P f\|_{\mathrm{D}(A)} \leq M\left(\left\|\varphi \cosh \left(\omega_{0} \cdot\right)\right\|_{p^{\prime}}+\left\|\varphi^{\prime} \cosh \left(\omega_{0} \cdot\right)\right\|_{p^{\prime}}\right)\|f\|_{1, p} .
$$

As $\mathcal{S}(X)$ is dense in $\mathrm{W}^{1, p}(X), P: \mathrm{W}^{1, p}(X) \rightarrow \mathrm{D}(A)$ is bounded.

Since $L_{\mu_{\omega}} \in \mathcal{L}\left(\mathrm{W}^{1, p}(X)\right)$, we can factorize $U_{\mu} \in \mathcal{L}(\mathrm{D}(A))$ as $U_{\mu}=P \circ L_{\mu_{\omega}} \circ \iota$ via bounded maps through $\mathrm{W}^{1, p}(X)$. Applying the real interpolation method with parameters $\theta$ and $q$ to the two factorizations of $U_{\mu}$, through $\mathrm{L}^{p}(X)$ respectively $\mathrm{W}^{1, p}(X)$, yields the commutative diagram of bounded maps

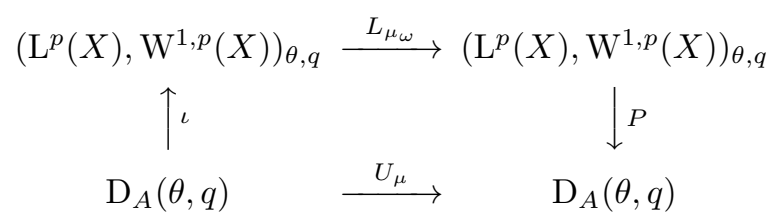

Finally, estimate the norms of $\iota$ and $P$ in this diagram by applying (1.5) to (3.1) and (3.3) respectively (3.2) and (3.4). This yields

$$
\left\|U_{\mu}\right\|_{\mathcal{L}\left(\mathrm{D}_{A}(\theta, q)\right)} \leq C^{\prime} M^{2}\left\|L_{\mu}\right\|_{\mathcal{L}\left(\left(\mathrm{L}^{p}(X), \mathrm{W}^{1, p}(X)\right)_{\theta, q}\right)}
$$

for a constant $C^{\prime} \geq 0$ independent of $\mu$. Now Lemma 2.5 concludes the proof.

3.2. Bounded groups. In this section we establish a version of the classical transference principle from [5] on interpolation spaces, already stated in the Introduction as Proposition 1.1. In the proof we use the convention $1 / \infty:=0$.

Proposition 3.2. Let $\theta \in(0,1), p \in[1, \infty)$ and $q \in[1, \infty]$. Then there exists a constant $C \geq 0$ such that the following holds. If $-\mathrm{i} A$ generate a $C_{0}$-group $(U(s))_{s \in \mathbb{R}}$ on a Banach space $X$ with $M:=\sup _{s \in \mathbb{R}}\|U(s)\|<\infty$, then

$$
\left\|\int_{\mathbb{R}} U(s) x \mu(\mathrm{d} s)\right\|_{\theta, q} \leq C M^{2}\left\|L_{\mu}\right\|_{\mathcal{L}\left(\mathrm{B}_{p, q}^{\theta}(X)\right)}\|x\|_{\theta, q}
$$

for all $\mu \in \mathrm{M}(\mathbb{R})$ and $x \in \mathrm{D}_{A}(\theta, q)$. 
Proof. First note that it suffices to establish (3.6) for measures with compact support. Indeed, approximating by measures with compact support then extends (3.6) to all $\mu \in \mathrm{M}(\mathbb{R})$. So fix $N>0$ and let $\mu \in \mathrm{M}(\mathbb{R})$ be such that $\operatorname{supp}(\mu) \subseteq[-N, N]$. We will factorize $U_{\mu}$ using the abstract transference principle from [13, Section 2]. To this end, let $\rho \in \mathrm{C}^{\infty}(\mathbb{R})$ be defined by

$$
\rho(s):= \begin{cases}c_{1} \exp \left(\frac{1}{s^{2}-1}\right) & |s|<1 \\ 0 & |s| \geq 1\end{cases}
$$

where $c_{1} \geq 0$ is such that $\int_{\mathbb{R}} \rho(s) \mathrm{d} s=1$. Fix $\alpha, \beta>0$ and define $\sigma(s):=\frac{1}{\alpha} \rho\left(\frac{s}{\alpha}\right)$ for $s \in \mathbb{R}$, and

$$
\psi:=\sigma * \mathbf{1}_{[-(N+3 \alpha+\beta), N+3 \alpha+\beta]} \quad \text { and } \quad \varphi:=\frac{1}{2(\alpha+\beta)} \sigma * \mathbf{1}_{[-(\alpha+\beta), \alpha+\beta]} .
$$

Then $\psi, \varphi \in \mathrm{C}^{\infty}(\mathbb{R})$ are such that $\operatorname{supp}(\varphi) \subseteq[-(2 \alpha+\beta), 2 \alpha+\beta]$,

$$
\psi \equiv 1 \text { on }[-(2 \alpha+N+\beta), 2 \alpha+N+\beta] \text { and } \int_{-(2 \alpha+\beta)}^{2 \alpha+\beta} \varphi(s) \mathrm{d} s=1 .
$$

Hence $\psi * \varphi \equiv 1$ on $[-N, N]$. Let $\iota: X \rightarrow \mathrm{L}^{p}(X)$ be given by

$$
\iota x(s):=\psi(-s) U(-s) x \quad(x \in X, s \in \mathbb{R}),
$$

and $P: \mathrm{L}^{p}(X) \rightarrow X$ by

$$
P f:=\int_{\mathbb{R}} \varphi(s) U(s) f(s) \mathrm{d} s \quad\left(f \in \mathrm{L}^{p}(X)\right) .
$$

Proposition 2.3 in [13] yields the factorization $U_{\mu}=P \circ L_{\mu} \circ \iota$, where we use that $(\psi * \varphi) \mu=\mu$. By Hölder's inequality,

$$
\|\iota\|_{\mathcal{L}\left(X, \mathrm{~L}^{p}(X)\right)} \leq M\|\psi\|_{p} \quad \text { and } \quad\|P\|_{\mathcal{L}\left(\mathrm{L}^{p}(X), X\right)} \leq M\|\varphi\|_{p^{\prime}}
$$

Moreover, $\iota: \mathrm{D}(A) \rightarrow \mathrm{W}^{1, p}(X)$ and $P: \mathrm{W}^{1, p}(X) \rightarrow \mathrm{D}(A)$ are bounded with

$$
\|\iota\|_{\mathcal{L}\left(\mathrm{D}(A), \mathrm{W}^{1, p}(X)\right)} \leq M\|\psi\|_{1, p} \quad \text { and } \quad\|P\|_{\mathcal{L}\left(\mathrm{W}^{1, p}(X), \mathrm{D}(A)\right)} \leq M\|\varphi\|_{1, p^{\prime}} .
$$

This follows by arguments almost identical to those in the proof of Proposition 3.1 Applying the real interpolation method with parameters $\theta$ and $q$ to the two factorizations of $U_{\mu}$, through $\mathrm{L}^{p}(X)$ and $\mathrm{W}^{1, p}(X)$, produces the commutative diagram of bounded maps

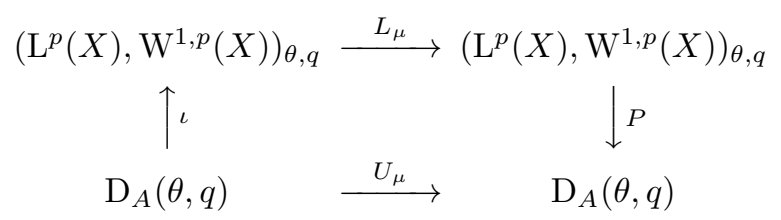

Use (1.5) on (3.7) and (3.8) to estimate the norms of $\iota$ and $P$ in this factorization as $\|\iota\| \leq M\|\psi\|_{1, p}$ and $\|P\| \leq M\|\varphi\|_{1, p^{\prime}}$. This yields

$$
\left\|U_{\mu}\right\|_{\mathcal{L}\left(\mathrm{D}_{A}(\theta, q)\right)} \leq M^{2}\|\psi\|_{1, p}\|\varphi\|_{1, p^{\prime}}\left\|L_{\mu}\right\|_{\mathcal{L}\left(\left(\mathrm{L}^{p}(X), \mathrm{W}^{1, p}(X)\right)_{\theta, q}\right)} .
$$

To determine $\|\psi\|_{1, p}$ and $\|\varphi\|_{1, p^{\prime}}$, note that

$$
\begin{aligned}
& \|\psi\|_{p} \leq\|\sigma\|_{1}\left\|\mathbf{1}_{[-(N+3 \alpha+\beta), N+3 \alpha+\beta]}\right\|_{p}=(2(N+3 \alpha+\beta))^{1 / p} \\
& \|\varphi\|_{p^{\prime}} \leq \frac{1}{2(\alpha+\beta)}\|\sigma\|_{1}\left\|\mathbf{1}_{[-(\alpha+\beta), \alpha+\beta]}\right\|_{p^{\prime}}=(2(\alpha+\beta))^{-1 / p}
\end{aligned}
$$


by Young's inequality. Since $\sigma$ is an even function that is decreasing on $[0, \alpha]$ and supported on $[-\alpha, \alpha]$, its derivative satisfies

$$
\left\|\sigma^{\prime}\right\|_{1}=-2 \int_{0}^{\alpha} \sigma^{\prime}(s) \mathrm{d} s=2(\sigma(0)-\sigma(\alpha))=\frac{2 \rho(0)}{\alpha} .
$$

Let $c_{2}:=2 \rho(0)$. Another application of Young's inequality yields

$$
\begin{aligned}
& \left\|\psi^{\prime}\right\|_{p} \leq\left\|\sigma^{\prime}\right\|_{1}\left\|\mathbf{1}_{[-(N+3 \alpha+\beta), N+3 \alpha+\beta]}\right\|_{p}=\frac{c_{2}}{\alpha}(2(N+3 \alpha+\beta))^{1 / p} \\
& \left\|\varphi^{\prime}\right\|_{p} \leq \frac{1}{2(\alpha+\beta)}\left\|\sigma^{\prime}\right\|_{1}\left\|\mathbf{1}_{[-(\alpha+\beta), \alpha+\beta]}\right\|_{p}=\frac{c_{2}}{\alpha}(2(\alpha+\beta))^{-1 / p}
\end{aligned}
$$

Hence (3.9) becomes

$$
\left\|U_{\mu}\right\|_{\mathcal{L}\left(\mathrm{D}_{A}(\theta, q)\right)} \leq M^{2}\left(1+\frac{c_{2}}{\alpha}\right)^{2}\left(\frac{N+3 \alpha+\beta}{\alpha+\beta}\right)^{1 / p}\left\|L_{\mu}\right\|_{\mathcal{L}\left(\left(\mathrm{L}^{p}(X), \mathrm{W}^{1, p}(X)\right)_{\theta, q}\right)} .
$$

Taking the infimum over $\alpha$ and $\beta$ yields

$$
\left\|U_{\mu}\right\|_{\mathcal{L}\left(\mathrm{D}_{A}(\theta, q)\right)} \leq M^{2}\left\|L_{\mu}\right\|_{\mathcal{L}\left(\left(\mathrm{L}^{p}(X), \mathrm{W}^{1, p}(X)\right)_{\theta, q}\right)} .
$$

Lemma 2.5 now establishes (3.6) and concludes the proof.

Remark 3.3. Note that the constant $C$ in Proposition 3.2 comes only from the equivalence of the norms on $\left(\mathrm{L}^{p}(X), \mathrm{W}^{1, p}(X)\right)_{\theta, q}$ and $\mathrm{B}_{p, q}^{\theta}(X)$, whereas in Proposition 3.1 a constant is present which is inherent to the transference method.

Remark 3.4. Let $p \in[1, \infty)$ and let $(U(s))_{s \in \mathbb{R}} \subseteq \mathcal{L}\left(\mathrm{L}^{p}(\mathbb{C})\right)$ be the shift group given by $U(s) f(t):=f(t+s)$ for $f \in \mathrm{L}^{p}(\mathbb{C}), s \in \mathbb{R}$ and almost all $t \in \mathbb{R}$. Then $(U(s)))_{s \in \mathbb{R}}$ is generated by $-\mathrm{i} A$, where $A f:=\mathrm{i} f^{\prime}$ for $f \in \mathrm{D}(A)=\mathrm{W}^{1, p}(\mathbb{C})$. Hence $\mathrm{D}_{A}(\theta, q)=\left(\mathrm{L}^{p}(\mathbb{C}), \mathrm{W}^{1, p}(\mathbb{C})\right)_{\theta, q}$ for $\theta \in(0,1)$ and $q \in[1, \infty]$. Moreover, for $\mu \in$ $\mathrm{M}(\mathbb{R})$ and $f \in \mathrm{L}^{p}(\mathbb{C})$,

$$
\int_{\mathbb{R}} U(s) f \mathrm{~d} \mu(s)=\mu * f=L_{\mu}(f) .
$$

Hence, with $U_{\mu}$ as in (2.2),

$$
\left\|U_{\mu}\right\|_{\mathcal{L}\left(\mathrm{D}_{A}(\theta, q)\right)}=\left\|L_{\mu}\right\|_{\mathcal{L}\left(\left(\mathrm{L}^{p}(\mathbb{C}), \mathrm{W}^{1, p}(\mathbb{C})\right)_{\theta, q}\right)} .
$$

This shows that (3.10) is sharp in general, up to possibly a change of constant. By Lemma 2.5. the same holds for (3.6).

Corollary 2.7 yields the following result, Corollary 1.2 from the Introduction.

Corollary 3.5. Let $\theta \in(0,1)$ and $q \in[1, \infty]$. Then there exists a constant $C \geq 0$ such that the following holds. Let $-\mathrm{i} A$ generate a $C_{0}$-group $(U(s))_{s \in \mathbb{R}}$ on a Banach space $X$ with $M:=\sup _{s \in \mathbb{R}}\|U(s)\|<\infty$, and let $\mu \in \mathrm{M}(\mathbb{R})$ be such that $\mathcal{F} \mu \in \mathrm{C}^{1}(\mathbb{R})$ with $\sup _{s \in \mathbb{R}}(1+|s|)\left|(\mathcal{F} \mu)^{\prime}(s)\right|<\infty$. Then

$$
\left\|\int_{\mathbb{R}} U(s) x \mu(\mathrm{d} s)\right\|_{\theta, q} \leq C M^{2} \sup _{s \in \mathbb{R}}|\mathcal{F} \mu(s)|+(1+|s|)\left|(\mathcal{F} \mu)^{\prime}(s)\right|\|x\|_{\theta, q}
$$

for all $x \in \mathrm{D}_{A}(\theta, q)$. 
Remark 3.6. To obtain Corollary 3.5 we used Corollary 2.7, but there are other ways to verify the conditions of Proposition 2.6, for instance Hörmander type assumptions, cf. 9. pp. 47-49]. More generally, one can define a norm on the space of all bounded Fourier multipliers $m$ on $\mathrm{B}_{p, q}^{r}(X)$ by $\|m\|_{\mathcal{M}\left(\mathrm{B}_{p, q}^{r}(X)\right)}:=\left\|T_{m}\right\|_{\mathcal{L}\left(\mathrm{B}_{p, q}^{r}(X)\right)}$, with $T_{m}$ as in (2.4). Proposition 3.2 yields $\left\|U_{\mu}\right\|_{\mathcal{L}\left(\mathrm{D}_{A}(\theta, q)\right)} \leq C\|\mathcal{F} \mu\|_{\mathcal{M}\left(\mathrm{B}_{p, q}^{r}(X)\right)}$, which cannot be improved in general, cf. Remark 3.4 .

Remark 3.7. If $X$ is a UMD space then (1.1) and the vector-valued Mikhlin multiplier theorem [10, Theorem E.6.2 b] yield an estimate

$$
\left\|\int_{\mathbb{R}} U(s) x \mu(\mathrm{d} s)\right\|_{X} \leq C M^{2}\|x\|_{X} \sup _{s \in \mathbb{R}}|\mathcal{F} \mu(s)|+\left|s(\mathcal{F} \mu)^{\prime}(s)\right|
$$

for all $x \in X$. Corollary 3.5 then follows from (1.5), and moreover singularities of $(\mathcal{F} \mu)^{\prime}$ at zero are allowed. However, in our setting of general Banach spaces, the inhomogeneity of the Besov space $\mathrm{B}_{p, q}^{r}(X)$ implies that a condition at zero on the multiplier is needed to deal with the term $\varphi_{0} m$ in Proposition 2.6.

Remark 3.8. Letting $f:=\mathcal{F} \mu$, Corollary 3.5 yields an estimate

$$
\left\|f\left(A_{\theta, q}\right)\right\| \leq C \sup _{s \in \mathbb{R}}|f(s)|+(1+|s|)\left|f^{\prime}(s)\right| .
$$

This is a functional calculus statement for $A_{\theta, q}$ involving functions on the real line. One may now ask to which functions $f$ on the real line the definition of $f\left(A_{\theta, q}\right)$ can be extended in a sensible manner such that (3.11) holds. We can take the closure of the Fourier transforms of measures in the space consisting of all functions $f \in \mathrm{C}^{1}(\mathbb{R})$ for which $\sup _{s \in \mathbb{R}}|f(s)|+(1+|s|)\left|f^{\prime}(s)\right|$ is finite, or approximate by holomorphic functions as in [18, Lemma 4.15], using Theorem 4.1. This will yield a definition of $f\left(A_{\theta, q}\right)$ for a class of functions on the real line and a bound as in (3.11), but the question then remains how this definition relates to other known extensions of functional calculi. In the present article we restrict ourselves to results about holomorphic functional calculi.

\section{Functional calculus Results}

We now use the theory established in the previous sections to prove our main functional calculus result, Theorem[1.3. Recall the definition of the analytic Mikhlin algebra $\mathrm{H}_{1}^{\infty}\left(\mathrm{St}_{\omega}\right)$ from (1.2).

Theorem 4.1. Let $-\mathrm{i} A$ be the generator of a $C_{0}$-group $(U(s))_{s \in \mathbb{R}}$ on a Banach space $X$ and let $\theta \in(0,1), q \in[1, \infty]$ and $\omega>\theta(U)$ be given. Then there exists a constant $C \geq 0$ such that $f\left(A_{\theta, q}\right) \in \mathcal{L}\left(\mathrm{D}_{A}(\theta, q)\right)$ with

$$
\left\|f\left(A_{\theta, q}\right)\right\|_{\mathcal{L}\left(\mathrm{D}_{A}(\theta, q)\right)} \leq C\|f\|_{\mathrm{H}_{1}^{\infty}\left(\mathrm{St}_{\omega}\right)}
$$

for all $f \in \mathrm{H}_{1}^{\infty}\left(\mathrm{St}_{\omega}\right)$. If $(U(s))_{s \in \mathbb{R}}$ is uniformly bounded then $C$ can be chosen independent of $\omega>0$.

Proof. First consider $f \in \mathrm{H}_{1}^{\infty}\left(\mathrm{St}_{\omega}\right) \cap \mathcal{E}\left(\mathrm{St}_{\omega}\right)$ and fix $\alpha \in(\theta(U), \omega)$ and $p \in[1, \infty)$. By Lemma 2.1 there exists $\mu \in \mathrm{M}_{\alpha}(\mathbb{R})$ such that $f=\mathcal{F} \mu$. By Lemmas 2.1 and 2.2 and Proposition 3.1 .

$$
\left\|f\left(A_{\theta, q}\right)\right\|=\left\|\left(U_{\mu}\right)_{\theta, q}\right\| \leq C_{1}\left\|L_{\mu_{\alpha}}\right\|_{\mathcal{L}\left(\mathrm{B}_{p, q}^{\theta}(X)\right)}=C_{1}\left\|T_{\mathcal{F} \mu_{\alpha}}\right\|_{\mathcal{L}\left(\mathrm{B}_{p, q}^{\theta}(X)\right)}
$$


for some constant $C_{1} \geq 0$, where $T_{\mathcal{F} \mu_{\alpha}}$ is as in (2.4). Since

$$
\mathcal{F} \mu_{\alpha}(s)=\frac{f(s+i \alpha)+f(s-i \alpha)}{2} \quad(s \in \mathbb{R}),
$$

Corollary 2.7 yields a constant $C_{2} \geq 0$ such that

$$
\left\|f\left(A_{\theta, q}\right)\right\| \leq C_{2} \sup _{s \in \mathbb{R}}\left|\mathcal{F} \mu_{\alpha}(s)\right|+(1+|s|)\left|\left(\mathcal{F} \mu_{\alpha}\right)^{\prime}(s)\right| \leq C_{2}\|f\|_{\mathrm{H}_{1}^{\infty}\left(\mathrm{St}_{\omega}\right)} .
$$

For general $f \in \mathrm{H}_{1}^{\infty}\left(\mathrm{St}_{\omega}\right)$ first assume that $q<\infty$. By part b) of Lemma 2.2. $\mathrm{D}\left(A_{\theta, q}\right)$ is dense in $\mathrm{D}_{A}(\theta, q)$. Let $\tau_{k}(z):=-k^{2}(i k-z)^{-2}$ for $k \in \mathbb{N}$ with $k>\omega$ and $z \in \mathrm{St}_{\omega}$. Then $\tau_{k}, f \tau_{k} \in \mathrm{H}_{1}^{\infty}\left(\mathrm{St}_{\omega}\right) \cap \mathcal{E}\left(\mathrm{St}_{\omega}\right)$,

$$
\sup _{k}\left\|f \tau_{k}\right\|_{\mathrm{H}_{1}^{\infty}\left(\mathrm{St}_{\omega}\right)} \leq\|f\|_{\mathrm{H}_{1}^{\infty}\left(\mathrm{St}_{\omega}\right)} \sup _{k}\left\|\tau_{k}\right\|_{\mathrm{H}_{1}^{\infty}\left(\mathrm{St}_{\omega}\right)}<\infty
$$

and $f \tau_{k}(z) \rightarrow f(z)$ as $k \rightarrow \infty$, for all $z \in \mathrm{St}_{\omega}$. Now (4.2) yields

$$
\left\|f \tau_{k}\left(A_{\theta, q}\right)\right\| \leq C_{2}\left\|f \tau_{k}\right\|_{\mathrm{H}_{1}^{\infty}\left(\mathrm{St}_{\omega}\right)} \leq C\|f\|_{\mathrm{H}_{1}^{\infty}\left(\mathrm{St}_{\omega}\right)}
$$

for some $C \geq 0$. Hence the Convergence Lemma 2.4 implies $f(A) \in \mathcal{L}(X)$ and

$$
\left\|f\left(A_{\theta, q}\right)\right\| \leq C\|f\|_{\mathrm{H}_{1}^{\infty}\left(\mathrm{St}_{\omega}\right)} .
$$

Finally, for $q=\infty$ the Reiteration Theorem [4, Theorem 3.5.3] yields

$$
\mathrm{D}_{A}(\theta, \infty)=\left(\mathrm{D}_{A}\left(\theta_{1}, 1\right), \mathrm{D}_{A}\left(\theta_{2}, 1\right)\right)_{\theta_{3}, \infty}
$$

with equivalence of norms, where $\theta_{1}, \theta_{2}, \theta_{3} \in(0,1)$ are such that $\theta_{1} \neq \theta_{2}$ and $\theta_{1}\left(1-\theta_{3}\right)+\theta_{2} \theta_{3}=\theta$. Combining (4.3) and (1.5) concludes the proof of the first statement.

In the case where $(U(s))_{s \in \mathbb{R}}$ is uniformly bounded, use Proposition 3.2 instead of 3.1 in (4.1) to obtain

$$
\left\|f\left(A_{\theta, q}\right)\right\| \leq C_{1}\left\|T_{\mathcal{F} \mu}\right\|_{\mathcal{L}\left(\mathrm{B}_{p, q}^{\theta}(X)\right)}
$$

for all $f \in \mathrm{H}_{1}^{\infty}\left(\mathrm{St}_{\omega}\right) \cap \mathcal{E}\left(\mathrm{St}_{\omega}\right)$ and some constant $C_{1} \geq 0$ independent of $\omega$. The rest of the proof is the same as before.

Remark 4.2. Compare Theorem 4.1 with Theorem 3.6 in 12 . There an estimate

$$
\|f(A)\|_{\mathcal{L}(X)} \leq C \sup _{z \in \mathrm{St}_{\omega}}|f(z)|+\left|z f^{\prime}(z)\right|
$$

is obtained when the underlying space $X$ is a UMD space, and the constant $C$ is independent of $\omega$ when the group in question is uniformly bounded. Theorem 4.1 follows from (4.4) by interpolation, and this seems to yield a stronger result since the term $\sup _{z \in \mathrm{St}_{\omega}}\left|f^{\prime}(z)\right|$ does not appear in (4.4). In fact, the norms $\sup _{z \in \mathrm{St}_{\omega}}|f(z)|+\left|z f^{\prime}(z)\right|$ and $\|f\|_{\mathrm{H}_{1}^{\infty}\left(\mathrm{St}_{\omega}\right)}$ are equivalent, since $0 \in \mathrm{St}_{\omega}$ for all $\omega>0$. So for generators of unbounded groups (4.4) does not yield an essentially better estimate than Theorem 4.1. This is different for generators of uniformly bounded groups, since the norm equivalence of $\sup _{z \in \mathrm{St}_{\omega}}|f(z)|+\left|z f^{\prime}(z)\right|$ and $\|f\|_{\mathrm{H}_{1}^{\infty}\left(\mathrm{St}_{\omega}\right)}$ fails as $\omega \downarrow 0$. Hence for generators of uniformly bounded groups (4.4) yields a strictly stronger result on $\mathrm{D}_{A}(\theta, q)$ than Theorem 4.1

Remark 4.3. Let $\lambda \in \mathbb{C}$ with $\operatorname{Re}(\lambda)>\omega$. By [10, Corollary 6.6.3], $\mathrm{D}\left((\lambda-\mathrm{i} A)^{\alpha}\right) \subseteq$ $\mathrm{D}_{A}(\alpha, \infty)$ for each $\alpha \in(0,1)$. Hence Theorem 4.1 yields $f(A)(\lambda-\mathrm{i} A)^{-\alpha} \in \mathcal{L}(X)$ for all $\omega>\theta(U), f \in \mathrm{H}_{1}^{\infty}\left(\mathrm{St}_{\omega}\right)$ and $\alpha>0$. However, this already follows from [3, Proposition 8.2.3] in a similar manner as in [15, Remark 5.2]. Moreover, using arguments as in [15, Remark 3.9], [3, Proposition 8.2.3] already implies that $f(A)$ : 
$\mathrm{D}_{A}(\theta, q) \rightarrow \mathrm{D}_{A}\left(\theta^{\prime}, q^{\prime}\right)$ is bounded for all $\theta^{\prime}<\theta$ and $q, q^{\prime} \in[1, \infty]$. The improvement that Theorem 4.1 provides lies in going from $\theta^{\prime}<\theta$ to $\theta^{\prime}=\theta$.

Remark 4.4. As already noted in Remark 3.6, we could have used Fourier multiplier results on Besov spaces other than Corollary 2.7 These lead to statements about the boundedness of functional calculi for other function algebras.

For $\varphi \in(0, \pi)$ define

$$
\mathrm{S}_{\varphi}:=\{z \in \mathbb{C}|| \arg (z) \mid<\varphi\},
$$

and for $\psi \in(0, \pi / 2)$ and $\omega>0$,

$$
\Sigma_{\psi}:=\mathrm{S}_{\psi} \cup-\mathrm{S}_{\psi}, \quad \mathrm{V}_{\psi, \omega}:=\mathrm{St}_{\omega} \cup \Sigma_{\psi} .
$$

Lemma 4.5. Let $\omega>\omega^{\prime}>0$ and $\psi \in(0, \pi / 2)$. Then $\mathrm{H}^{\infty}\left(\mathrm{V}_{\omega, \psi}\right)$ is continuously embedded in $\mathrm{H}_{1}^{\infty}\left(\mathrm{St}_{\omega^{\prime}}\right)$.

Proof. This follows in a straightforward manner from Lemma 4.5 in [12.

Corollary 4.6. Let $-\mathrm{i} A$ be the generator of a $C_{0}$-group $(U(s))_{s \in \mathbb{R}}$ on a Banach space $X$ and let $\theta \in(0,1)$ and $q \in[1, \infty]$. Then $A_{\theta, q}$ has a bounded $\mathrm{H}^{\infty}\left(\mathrm{V}_{\omega, \psi}\right)$ calculus for all $\omega>\theta(U)$ and $\psi \in(0, \pi / 2)$.

So far we have considered functional calculus on interpolation spaces for the couple $(X, \mathrm{D}(A))$. The next corollary extends our results to other interpolation couples.

Corollary 4.7. Let $-\mathrm{i} A$ be the generator of a $C_{0}$-group $(U(s))_{s \in \mathbb{R}}$ on a Banach space $X$ and let $\theta \in(0,1), q \in[1, \infty]$ and $m, n \in \mathbb{N}_{0}$ with $m \neq n$. Then the part of $A$ in $\left(\mathrm{D}\left(A^{m}\right), \mathrm{D}\left(A^{n}\right)\right)_{\theta, q}$ has a bounded $\mathrm{H}_{1}^{\infty}\left(\mathrm{St}_{\omega}\right)$-calculus for all $\omega>\theta(U)$. If $(U(s))_{s \in \mathbb{R}}$ is uniformly bounded then the constant bounding the calculus is independent of $\omega>0$.

Proof. First note that since

$$
\left(\mathrm{D}\left(A^{m}\right), \mathrm{D}\left(A^{n}\right)\right)_{\theta, q}=\left(\mathrm{D}\left(A^{n}\right), \mathrm{D}\left(A^{m}\right)\right)_{1-\theta, q}
$$

by [4, Theorem 3.4.1], we may assume that $m<n$. Using the similarity transform $R(\lambda, A)^{m}: X \rightarrow \mathrm{D}\left(A^{m}\right)$, it suffices to let $m=0$. Suppose that $n \theta \notin \mathbb{N}$. By Lemma 3.1.3 and Proposition 3.1.8 in [19,

$$
\left(X, \mathrm{D}\left(A^{n}\right)\right)_{\theta, q}=\left(\mathrm{D}\left(A^{k}\right), \mathrm{D}\left(A^{k+1}\right)\right)_{\theta^{\prime}, q}
$$

for some $k \in \mathbb{N}_{0}$ and $\theta^{\prime} \in(0,1)$. Another similarity transform shows that we can let $k=0$. Now Theorem 4.1 yields the statement.

If $k:=n \theta \in \mathbb{N}$, the Reiteration Theorem [4, Theorem 3.5.3] yields

$$
\left(X, \mathrm{D}\left(A^{n}\right)\right)_{\theta, q}=\left(\left(\mathrm{D}\left(A^{k-1}\right), \mathrm{D}\left(A^{k}\right)\right)_{1 / 2, q},\left(\mathrm{D}\left(A^{k}\right), \mathrm{D}\left(A^{k+1}\right)\right)_{1 / 2, q}\right)_{1 / 2, q} .
$$

By what we have already shown and (1.5), this concludes the proof. 


\section{Additional Results}

We now deduce several applications of Theorem 4.1. Corollary 4.7 can be applied in this section to yield results for other interpolation couples.

We first state a proposition about the convergence of certain principal value integrals, an interpolation version of [12, Theorem 4.4] on general Banach spaces. If $g \in \mathrm{L}^{1}[-1,1]$ is even then by $\mathrm{PV}-g(s) / s$ we mean the distribution defined by

$$
\langle\mathrm{PV}-g(s) / s, \varphi\rangle:=\lim _{\epsilon \searrow 0} \int_{\epsilon \leq|s| \leq 1} g(s) \varphi(s) \frac{\mathrm{d} s}{s}
$$

for $\varphi \in \mathrm{C}^{\infty}(\mathbb{R})$ compactly supported. By $\mathrm{BV}[-1,1]$ we denote the functions of bounded variation on $[-1,1]$.

Proposition 5.1. Let $-\mathrm{i} A$ be the generator of a $C_{0}-$ group $(U(s))_{s \in \mathbb{R}}$ on a Banach space $X$. Let $g \in \mathrm{BV}[-1,1]$ be even and set $f:=\mathcal{F}(\mathrm{PV}-g(s) / s)$. Then $f\left(A_{\theta, q}\right) \in$ $\mathcal{L}\left(\mathrm{D}_{A}(\theta, q)\right)$ and

$$
f(A) x=\lim _{\epsilon \searrow 0} \int_{\epsilon \leq|s| \leq 1} g(s) U(s) x \frac{\mathrm{d} s}{s}
$$

for all $\theta \in(0,1), q \in[1, \infty)$ and $x \in \mathrm{D}_{A}(\theta, q)$.

Proof. By [12, Lemma 4.3], $f \in \mathrm{H}_{1}^{\infty}\left(\mathrm{St}_{\omega}\right)$ for all $\omega>0$. Theorem 4.1 now yields the first statement. For (5.1) we may let $q<\infty$, since $\mathrm{D}_{A}(\theta, \infty) \subseteq \mathrm{D}_{A}\left(\theta^{\prime}, 1\right)$ for $\theta^{\prime}<\theta$ [19, Proposition 1.1.4]. Now use the Convergence Lemma as in the proof of [12, Theorem 4.4].

Remark 5.2. Convergence in (5.1) takes place in $\mathrm{D}_{A}(\theta, q)$ for $q<\infty$. For $q=\infty$ the limit and the integral converge in $X$ and in $\mathrm{D}_{A}\left(\theta^{\prime}, q^{\prime}\right)$ for $\theta^{\prime}<\theta$ and $q^{\prime} \in[1, \infty)$. Compare with Remark 2.3.

5.1. Results for sectorial operators and cosine functions. An operator $A$ on a Banach space $X$ is sectorial of angle $\varphi \in(0, \pi)$ if $\sigma(A) \subseteq \overline{\mathrm{S}_{\varphi}}$, where $\mathrm{S}_{\varphi}$ is as in (4.5), and if $\sup \left\{\|z R(z, A)\| \mid \psi \in \mathbb{C} \backslash \mathrm{S}_{\psi}\right\}<\infty$ for all $\psi \in(\varphi, \pi)$. A functional calculus for sectorial operators can be constructed by a method similar to the one used for strip-type operators. For details see [10, Chapter 2].

If $A$ is an injective sectorial operator of angle $\varphi \in(0, \pi)$ then $\log (A)$ is defined, as is $f(A)$ for all $f \in \mathrm{H}^{\infty}\left(\mathrm{S}_{\psi}\right)$ and $\psi \in(\varphi, \pi)$. A sectorial operator $A$ has bounded imaginary powers if $A$ is injective and if $-\mathrm{i} \log (A)$ is the generator of a $C_{0}$-group $(U(s))_{s \in \mathbb{R}}$ on $X$. Then $U(s)=A^{-\mathrm{i} s}$ for all $s \in \mathbb{R}$, and we write $A \in \operatorname{BIP}(X)$. Moreover, $A$ is sectorial of angle $\theta_{A}:=\theta(U)$, by [10, Corollary 4.3.4].

For $\psi \in(0, \pi)$ define $\mathrm{H}_{\log }^{\infty}\left(\mathrm{S}_{\psi}\right)$ to be the unital Banach algebra of all $f \in \mathrm{H}^{\infty}\left(\mathrm{S}_{\psi}\right)$ for which

$$
\|f\|_{\mathrm{H}_{\mathrm{log}}^{\infty}\left(\mathrm{S}_{\psi}\right)}:=\sup _{z \in \mathrm{S}_{\psi}}|f(z)|+(1+|\log (z)|)\left|z f^{\prime}(z)\right|<\infty,
$$

endowed with the norm $\|\cdot\|_{\mathrm{H}_{\log }^{\infty}\left(\mathrm{S}_{\psi}\right)}$.

Proposition 5.3. Let $X$ be a Banach space and $A \in \operatorname{BIP}(X)$ such that $\theta_{A}<\pi$. Let $\theta \in(0,1)$ and $q \in[1, \infty]$. Set $Y:=(X, \mathrm{D}(\log (A)))_{\theta, q}$. Then $A_{Y}$ has a bounded $\mathrm{H}_{\log }^{\infty}\left(\mathrm{S}_{\psi}\right)$-calculus on $Y$ for all $\psi \in\left(\theta_{A}, \pi\right)$. If $\sup _{s \in \mathbb{R}}\left\|A^{\mathrm{i} s}\right\|<\infty$ then the constant bounding the calculus is independent of $\psi>0$. 
Proof. Let $\psi \in\left(\theta_{A}, \pi\right)$ be given and note that $f \mapsto f \circ \log$ is an isometric algebra isomorphism $\mathrm{H}_{1}^{\infty}\left(\mathrm{St}_{\psi}\right) \rightarrow \mathrm{H}_{\log }^{\infty}\left(\mathrm{S}_{\psi}\right)$. By Lemma 2.2 as well as Theorem 4.2.4 and Proposition 6.1.2 from [10],

$$
f\left(\log (A)_{Y}\right)=f(\log (A))_{Y}=(f \circ \log )(A)_{Y}=(f \circ \log )\left(A_{Y}\right)
$$

for all $f \in \mathrm{H}_{1}^{\infty}\left(\mathrm{St}_{\psi}\right)$. Now Theorem 4.1 concludes the proof.

Remark 5.4. Let $A$ be an injective sectoral operator of angle $\varphi \in(0, \pi)$, and let $\alpha>0, \theta \in(0,1)$ and $q \in[1, \infty]$. By [10, Corollary 6.6.3], a special case of which was proved by Dore [7, Theorem 3.2], the part of $A$ in $\left(X, \mathrm{D}\left(A^{\alpha}\right) \cap \mathrm{R}\left(A^{\alpha}\right)\right)_{\theta, q}$ has a bounded $\mathrm{H}^{\infty}\left(\mathrm{S}_{\psi}\right)$-calculus for all $\psi \in(\varphi, \pi)$. Here $\mathrm{R}(A)$ is the range of $A$. By [10, Corollary 6.6.3] and because $\log (A) A^{\alpha \theta}(1+A)^{-2 \alpha \theta} \in \mathcal{L}(X)$,

$$
\left(X, \mathrm{D}\left(A^{\alpha}\right) \cap \mathrm{R}\left(A^{\alpha}\right)\right)_{\theta, q} \subseteq\left(X, \mathrm{D}\left(A^{\alpha}\right)\right)_{\theta, q} \subseteq \mathrm{D}\left(A^{\alpha \theta}\right) \subseteq \mathrm{D}(\log (A)),
$$

and in general $\mathrm{D}(\log (A))$ is strictly included in $(X, \mathrm{D}(\log (A)))_{\theta^{\prime}, q^{\prime}}$ for all $\theta^{\prime} \in(0,1)$ and $q^{\prime} \in[1, \infty]$. Hence the result of Dore does not imply Proposition 5.3

A cosine function $\operatorname{Cos}: \mathbb{R} \rightarrow \mathcal{L}(X)$ on a Banach space $X$ is a strongly continuous mapping such that $\operatorname{Cos}(0)=I$ and

$$
\operatorname{Cos}(t+s)+\operatorname{Cos}(t-s)=2 \operatorname{Cos}(t) \operatorname{Cos}(s)
$$

for all $s, t \in \mathbb{R}$. Then

$$
\theta(\operatorname{Cos}):=\inf \left\{\omega \geq 0 \mid \exists M \geq 0:\|\operatorname{Cos}(t)\| \leq M \mathrm{e}^{\omega|t|} \text { for all } t \in \mathbb{R}\right\}<\infty .
$$

The generator of a cosine function is the unique operator $-A$ on $X$ that satisfies

$$
\lambda R\left(\lambda^{2},-A\right)=\int_{0}^{\infty} \mathrm{e}^{-\lambda t} \operatorname{Cos}(t) \mathrm{d} t
$$

for $\lambda>\theta(\mathrm{Cos})$. Then $A$ is an operator of parabola-type $\omega=\theta(\mathrm{Cos})$. This means that $\sigma(A) \subseteq \overline{\Pi_{\omega}}$, where $\Pi_{\omega}:=\left\{z^{2} \mid z \in \mathrm{St}_{\omega}\right\}$, and that for all $\omega^{\prime}>\omega$ there exists $M_{\omega^{\prime}} \geq 0$ such that

$$
\|R(\lambda, A)\| \leq \frac{M_{\omega^{\prime}}}{\sqrt{|\lambda|}\left(|\operatorname{Im}(\sqrt{\lambda})|-\omega^{\prime}\right)}
$$

for all $\lambda \notin \Pi_{\omega^{\prime}}$. For such operators there is a natural functional calculus, as before, and a version of Lemma 2.2 holds. For details see [14]. For $\omega>0$ let

$$
\mathrm{H}_{1}^{\infty}\left(\Pi_{\omega}\right):=\left\{f \in \mathrm{H}^{\infty}\left(\Pi_{\omega}\right)\left|\|f\|_{\mathrm{H}_{1}^{\infty}\left(\Pi_{\omega}\right)}:=\sup _{z \in \Pi_{\omega}}\right| f(z)|+(1+|z|)| f^{\prime}(z) \mid<\infty\right\},
$$

a Banach algebra when endowed with the norm $\|\cdot\|_{\mathrm{H}_{1}^{\infty}\left(\Pi_{\omega}\right)}$.

Proposition 5.5. Let $-A$ be the generator of a cosine function $\operatorname{Cos}$ on a Banach space $X$ and let $\theta \in(0,1), q \in[1, \infty]$. Then the part $A_{\theta, q}$ of $A$ in $\mathrm{D}_{A}(\theta, q)$ has a bounded $\mathrm{H}_{1}^{\infty}\left(\Pi_{\omega}\right)$-calculus for all $\omega>\theta(\operatorname{Cos})$. If $\sup _{s \in \mathbb{R}}\|\operatorname{Cos}(s)\|<\infty$ then the constant bounding the calculus is independent of $\omega>0$.

Proof. We mainly follow [12, Theorem 5.3], providing extra details where necessary. There is a unique subspace $V \subseteq X$, the Kisyński space, such that the operator $-\mathrm{i} \mathcal{A}$,

$$
\mathcal{A}:=\mathrm{i}\left[\begin{array}{cc}
0 & \mathrm{I}_{V} \\
-A & 0
\end{array}\right],
$$


with domain $\mathrm{D}(\mathcal{A}):=\mathrm{D}(A) \times V$, generates a $C_{0}$-group $(U(s))_{s \in \mathbb{R}}$ on $X \times V$. Moreover, $\theta(\operatorname{Cos})=\theta(U)$ [11, Theorem 6.2].

Let $\omega>\theta(\operatorname{Cos})$. Then $f \in \mathrm{H}^{\infty}\left(\Pi_{\omega}\right)$ is an element of $\mathrm{H}_{1}^{\infty}\left(\Pi_{\omega}\right)$ if and only if $\left[z \mapsto g(z):=f\left(z^{2}\right)\right] \in \mathrm{H}_{1}^{\infty}\left(\mathrm{St}_{\omega}\right)$, with $\|g\|_{\mathrm{H}_{1}^{\infty}\left(\mathrm{St}_{\omega}\right)} \leq 4\|f\|_{\mathrm{H}_{1}^{\infty}\left(\Pi_{\omega}\right)}$. Moreover, $f(A) \oplus f\left(A_{V}\right)=g(\mathcal{A})$ and

$$
f\left(A_{\theta, q}\right) \oplus f\left(A_{V}\right)=\left(f(A) \oplus f\left(A_{V}\right)\right)_{Y}=g(\mathcal{A})_{Y}=g\left(\mathcal{A}_{Y}\right)
$$

for all $f \in \mathrm{H}_{1}^{\infty}\left(\Pi_{\omega}\right)$, where $Y:=\mathrm{D}_{\mathcal{A}}(\theta, q)=\mathrm{D}_{A}(\theta, q) \times V$. Hence Theorem 4.1 concludes the proof.

Acknowledgements. We would like to thank our colleagues for interesting discussions and helpful ideas.

\section{REFERENCES}

[1] H. Amann. Operator-valued Fourier multipliers, vector-valued Besov spaces, and applications. Math. Nachr., 186:5-56, 1997.

[2] W. Arendt. Semigroups and evolution equations: Functional calculus, regularity and kernel estimates. In C.M.Dafermos E. Feireisl, editor, Handbook of Differential Equations, pages 1-85. Elsevier/North Holland, Amsterdam, 2004.

[3] W. Arendt, C.J.K. Batty, M. Hieber, and F. Neubrander. Vector-valued Laplace transforms and Cauchy problems, volume 96 of Monographs in Mathematics. Birkhäuser/Springer Basel AG, Basel, second edition, 2011.

[4] J. Bergh and J. Löfström. Interpolation spaces. An introduction. Springer-Verlag, Berlin, 1976. Grundlehren der Mathematischen Wissenschaften, No. 223.

[5] E. Berkson, T.A. Gillespie, and P.S. Muhly. Generalized analyticity in UMD spaces. Ark. Mat., 27(1):1-14, 1989.

[6] G. Dore. $H^{\infty}$ functional calculus in real interpolation spaces. Studia Math., 137(2):161-167, 1999.

[7] G. Dore. $H^{\infty}$ functional calculus in real interpolation spaces. II. Studia Math., 145(1):75-83, 2001.

[8] K. Engel and R. Nagel. One-parameter semigroups for linear evolution equations, volume 194 of Graduate Texts in Mathematics. Springer-Verlag, New York, 2000. With contributions by S. Brendle, M. Campiti, T. Hahn, G. Metafune, G. Nickel, D. Pallara, C. Perazzoli, A. Rhandi, S. Romanelli and R. Schnaubelt.

[9] M. Girardi and L. Weis. Operator-valued Fourier multiplier theorems on Besov spaces. Math. Nachr., 251:34-51, 2003.

[10] M. Haase. The functional calculus for sectorial operators, volume 169 of Operator Theory: Advances and Applications. Birkhäuser Verlag, Basel, 2006.

[11] M. Haase. Functional calculus for groups and applications to evolution equations. J. Evol. Equ., 7(3):529-554, 2007.

[12] M. Haase. A transference principle for general groups and functional calculus on UMD spaces. Math. Ann., 345(2):245-265, 2009.

[13] M. Haase. Transference principles for semigroups and a theorem of Peller. J. Fun. Anal., 261(10):2959-2998, 2011.

[14] M. Haase. The functional calculus approach to cosine operator functions. In Recent Trends in Analysis. Proceedings of the Conference in honor of N.K. Nikolski held in Bordeaux 2011, pages 123-147. Theta Foundation, 2013.

[15] M. Haase and J. Rozendaal. Functional calculus for semigroup generators via transference. J. Funct. Anal., 265(12):3345-3368, 2013.

[16] T. Hytönen. Fourier embeddings and Mihlin-type multiplier theorems. Math. Nachr., 274/275:74-103, 2004

[17] N.J. Kalton and L. Weis. The $H^{\infty}$-calculus and sums of closed operators. Math. Ann., 321(2):319-345, 2001.

[18] C. Kriegler. Spectral multipliers, R-bounded homomorphisms, and analytic diffusion semigroups. $\mathrm{PhD}$ thesis, Karlsruhe Institute of Technology, 2009. Online at http://digbib.ubka.uni-karlsruhe.de/volltexte/1000015866 
[19] A. Lunardi. Interpolation theory. Appunti. Scuola Normale Superiore di Pisa (Nuova Serie). [Lecture Notes. Scuola Normale Superiore di Pisa (New Series)]. Edizioni della Normale, Pisa, second edition, 2009.

Delft Institute of Applied Mathematics, Mekelweg 4, 2628CD Delft, The NetherLANDS

E-mail address: m.h.a.haase@tudelft.nl

Delft Institute of Applied Mathematics, Mekelweg 4, 2628CD Delft, The NetherLANDS

E-mail address, corresponding author: janrozendaalmath@gmail.com 